\title{
Water Flow in Silica Nanopores Coated by Carbon Nanotubes from a Wetting Translucency Perspective
}

Wagemann, Enrique; Walther, Jens H.; Cruz-Chu, Eduardo R.; Zambrano, Harvey A.

Published in:

Journal of Physical Chemistry C

Link to article, DOI:

10.1021/acs.jpcc.9b05294

Publication date:

2019

Document Version

Peer reviewed version

Link back to DTU Orbit

Citation (APA):

Wagemann, E., Walther, J. H., Cruz-Chu, E. R., \& Zambrano, H. A. (2019). Water Flow in Silica Nanopores Coated by Carbon Nanotubes from a Wetting Translucency Perspective. Journal of Physical Chemistry C, 123, 2563525642. https://doi.org/10.1021/acs.jpcc.9b05294

\section{General rights}

Copyright and moral rights for the publications made accessible in the public portal are retained by the authors and/or other copyright owners and it is a condition of accessing publications that users recognise and abide by the legal requirements associated with these rights.

- Users may download and print one copy of any publication from the public portal for the purpose of private study or research.

- You may not further distribute the material or use it for any profit-making activity or commercial gain

- You may freely distribute the URL identifying the publication in the public portal 


\section{Water Flow in Silica Nanopores Coated by}

\section{Carbon Nanotubes from a Wetting}

\section{Translucency Perspective}

Enrique Wagemann, ${ }^{\dagger}$ Jens H. Walther, ${ }^{\ddagger}$ Eduardo R. Cruz-Chú, ${ }^{\S}$ and Harvey A. Zambrano*,\|

Facultad de Ingeniería, Universidad de Concepción, Concepción, Chile, Technical University of Denmark, Copenhagen, Denmark, Chair of Computational Science, ETH

Zurich, Zurich, Switzerland, Laboratorios de Investigación y Desarrollo, Facultad de Ciencias y Filosofía, Universidad Peruana Cayetano Heredia, Lima, Perú, and Department of Mechanical Engineering, Universidad Técnica Federico Santa María, Valparaíso, Chile

E-mail: harvey.zambrano@usm.cl

\footnotetext{
${ }^{*}$ To whom correspondence should be addressed

†Universidad de Concepción

‡Technical University of Denmark

『ETH Zurich

$\S$ Universidad Peruana Cayetano Heredia

"Universidad Técnica Federico Santa María
} 


\begin{abstract}
Nearly frictionless water transport makes carbon nanotubes promising materials for use as conduits in nanofluidic applications. Here, we conduct Molecular Dynamics simulations of water flow within amorphous silica nanopores coated by a $(39,39)$ Single Walled Carbon Nanotube (SWCNT). Our atomistic models describe the interaction between water and pore walls based on two possible scenarios, translucency and opacity to wetting of a SWCNT. Simulation results indicate that the SWCNT coating enhances water flow through silica pores ca. 10 times compared to predictions from classical Hagen-Poiseuille relation. By varying the strength of the water-pore interaction, we study the relationship between surface wettability and hydrodynamic slippage. We observe an increase in the slip length for higher values of the water contact angle. Moreover, cases with SWCNT opacity and translucency to wetting display a substantial difference in the computed slippage, showing that the water contact angle is not the only factor that determines the slip boundary condition under nanoconfinement. We attribute this disparity to the corrugation of the potential energy landscape at the inner pore wall. The present study provides a theoretical framework for the use of carbon nanotube-based coatings to design more efficient nanofluidic conduits.
\end{abstract}

\title{
Introduction
}

Recent advances in experimental techniques ${ }^{1-4}$ have made possible the fabrication of nanofluidic systems capable to manipulate fluids with unprecedented control and efficiency. ${ }^{5-9}$ Hence, water transport in nanoconfined geometries is of great scientific and practical interest, due to its potential application in a wide range of technologies, such as water desalination, ${ }^{10,11}$ Lab-On-a-Chip, ${ }^{12}$ nanomedicine ${ }^{13}$ and DNA sequencing. ${ }^{14}$ As a result of the extremely large ratio between surface area and confined volume, the hydrodynamic properties of a nanoconfined fluid are strongly influenced by interfacial phenomena. ${ }^{15-17}$ Therefore, for hydrophilic nanochannels, fluid flows are generally associated with very large viscous re- 
sistance. ${ }^{18}$ In this context, the design of functional nanofluidic devices and nanostructured membranes requires effective strategies to reduce hydrodynamic losses.

Carbon nanotubes (CNT) are nanostructured carbon allotropes that have attracted significant attention due to their remarkable physical and chemical properties. ${ }^{19,20}$ In the last decade, numerous studies have reported that the flow rates within carbon nanotubes are several orders of magnitude higher than those measured in hydrophilic conduits with similar diameter. ${ }^{11,21-26}$ This ultra-low resistance to water flow, alongside their proved high mechanical strength and chemical stability, make CNTs ideal candidates to develop novel membranes ${ }^{11,20,27}$ and to be used as fluid conduits in integrated nanofluidic devices. ${ }^{7}$ In particular, the low friction of water in contact with CNTs has been associated with the exceptional atomic smoothness of their graphitic walls ${ }^{22,28,29}$ and the weak interaction between water molecules and CNT walls. ${ }^{16,23,25}$ Nevertheless, recent reports on the effect of airborne contaminants ${ }^{30}$ and the effect of the underlying substrates ${ }^{31-33}$ on the wettability of supported graphene (GE), have put in the spotlight our current understanding of the interaction of water with graphitic materials. Indeed, contradictory reports on how GE monolayers modify the wettability of coated substrates have aroused intense debate, in particular about the influence of an atomically thin material on the interaction of water with the underlying substrate. Those studies report the influence of GE coating to be from complete wetting transparency ${ }^{31}$ to complete wetting opacity ${ }^{34,35}$ to the coated substrate. In particular, Shih et al. ${ }^{32,33}$ suggest that the equilibrium separation distance between a fluid and the underlying substrate, induced by the atomically thin wall of a GE coating, is sufficiently short to allow the direct interaction between water molecules and substrate atoms. Hence, GE is denoted as a semi-transparent, i.e., translucent, coating. Following their results, it can be inferred that other sufficiently thin coating materials could reproduce this reported phenomenon of translucency to wettability. Note that an open-ended SWCNT is essentially a GE sheet rolled into a cylinder, ${ }^{19,36}$ therefore in the present study, we use atomistic simulations to probe the SWCNT translucency to wettability and evaluate its effect on the transport properties 
of water confined inside cylindrical hydrophilic silica pores.

In two recent studies, Hong et al. ${ }^{37}$ and Ashraf et al. ${ }^{38}$ studied the effect of applied electrical voltages and chemical functionalization on the wettability of substrates coated with GE. Their results show that the Water Contact Angle (WCA) of these surfaces can be tuned by applying chemical doping or imposing electrical voltages. Moreover, they suggest that this tunability is a consequence of shift in the GE Fermi level, resulting in an effective modification of the interaction strength between GE and water. In the present work, we take into consideration these factors by simulating water flow within a silica nanopore coated by a coaxial SWCNT, with an increasing strength of the interaction between CNT atoms and water molecules. In this study, Molecular Dynamics (MD) simulations are carried out to investigate the use of a SWCNT as wall coating in a hydrophilic silica nanopore. A snapshot of the studied system is presented in Figure 1. In our simulations, the interactions between water molecules and the pore atoms are modeled to reproduce two possible scenarios; the wetting translucency reported by Shih et al. ${ }^{32,33}$ and the tunnability of the wetting reported by Hong et al. ${ }^{37}$ and Ashraf et al. ${ }^{38}$ We evaluate the hydrodynamic properties of water confined within the pore considering different degrees of wettability. To this end, we conduct nonequilibrium MD (NEMD) simulations of Poiseuille-like flow. Our results show that, despite reproducing the same wettability, a substantial difference in the hydrodynamic slippage is found when comparing both scenarios. We associate this disparity to the different energy landscapes computed within the coated pore for each scenario. Following, we provide quantitative evidence that a SWCNT coating the inner surface of a silica nanopore results in substantial flow enhancement of nanoconfined water. 


\section{Computational methods}

\section{Simulation details}

The MD simulations are carried out using the parallel MD package FASTTUBE, which has been extensively used to study liquids confined inside CNTs, GE layers and silica channels. ${ }^{39-45}$ The leap-frog scheme is used to integrate the equations of motion, with an integration time step of $2 \mathrm{fs}$. Temperature control is achieved by employing a Berendsen heat bath. ${ }^{46}$ An orthorhombic simulation box is used, with dimensions chosen to match the ones of the pore. Periodic boundary condition are enforced in all directions. Van der Walls interactions are truncated at $1 \mathrm{~nm}$. To account for the electrostatic interactions between silica atoms, in order to reduce computational cost, we employ a shifted Coulombic potential with a cut-off of $1 \mathrm{~nm}$. A Smoothed Particle Mesh Ewald (SPME) algorithm ${ }^{47}$ with a real-space cutoff of $1.0 \mathrm{~nm}$ and mesh spacing of $0.125 \mathrm{~nm}$ is employed to compute the long range Coulombic electrostatics between water molecules interacting with each other and for silica atoms interacting with water molecules.

In our simulations, the rigid $\mathrm{SPC} / \mathrm{E} \mathrm{model}^{48}$ is employed to describe water molecules. The carbon-carbon valence forces within the CNT are described using Morse, harmonic angle and torsion potentials. ${ }^{42,49}$ The CNT-silica interactions are described employing a 126 Lennard-Jones potential with parameters from Zhang and Li. ${ }^{50}$ The interactions between silica atoms are described using the TTAMm potential developed by Guissani and Guillot. ${ }^{51}$ In order to maintain the internal silica substrate active while avoiding the deformation of the pore, silica atoms interact with each other by employing the original partial charges of the TTAM potential $\left(q_{\mathrm{Si}}=2.4 e\right.$ and $\left.q_{\mathrm{O}_{\mathrm{SiO}_{2}}}=-1.2 e\right)$, whereas silica atoms and water molecules

interact through a set of lower partial charges $\left(q_{\mathrm{Si}}=1.3 e\right.$ and $\left.q_{\mathrm{O}_{\mathrm{SiO}_{2}}}=-0.65 e\right) .{ }^{52,53}$ Further details of the employed models are presented in the Supporting Information. 


\section{Pore model}

Our system consists of water confined inside an amorphous silica nanopore internally coated by a $(39,39)$ armchair SWCNT, as shown in Figure 1 . The amorphous silica substrate is obtained from a thermal annealing protocol described in the Supporting Information. An internal radius of $3.155 \mathrm{~nm}$ with a standard deviation of $0.285 \mathrm{~nm}$ is computed for the silica nanopore, whereas the CNT within the pore has a radius of $2.6209 \mathrm{~nm}$ with a standard deviation of $0.065 \mathrm{~nm}$. The contact distance between the CNT and the surface of the underlying substrate is $0.405 \mathrm{~nm}$ with a standard deviation of $0.0854 \mathrm{~nm}$. Comparing the standard deviation of the contact distance with the standard deviation of the radii, we infer that the CNT does not fully inherit the roughness of the underlying silica, thus, the internal SWCNT coating reduces the roughness of the pore to approximately a $25 \%$ of its original value for the pristine pore. In our simulations, removal of viscous heating is achieved by extracting heat through the walls. To this end, the silica atoms near the inner pore surface and the carbon atoms of the CNT are coupled to a Berendsen thermostat, with a coupling constant of $0.005 \mathrm{ps}$. The positions of the atoms in the bulk silica are maintained fixed during the entire simulations.

\section{Pore-Water interactions}

The employed models to describe the interaction between confined water and the solid surface reproduce two scenarios that portray similar wettabilities, but differ in the way that water molecules interact with the coated pore. In the first scenario, CNT is translucent to the wettability of the underlying silica, as a result of its atomically thin wall. The interaction between water and the coated silica pore is therefore modeled as the sum of two interactions; water-CNT and water-silica. All the cases that model this scenario will be denominated as "translucency cases". In the second scenario, the underlying silica substrate influences only

indirectly the water transport by altering the strength of the CNT-water interaction and imposing a physical corrugation along the CNT wall. Thus, the water behavior within the 
coated pore in this scenario, is exclusively the result of the interplay between the CNT atoms and the water molecules. The cases that model this scenario will be denoted as "opacity cases".

In both scenarios, the water-CNT interactions are described using a 12-6 Lennard-Jones potential between $\mathrm{O}$ and $\mathrm{C}$ atoms. In the translucency cases, we employ the parameters of Werder et al., ${ }^{41}$ that were calibrated to recover a macroscopic WCA of $86^{\circ}$ on graphite. The values of these parameters are $\sigma_{\mathrm{C}-\mathrm{O}_{\mathrm{H}_{2} \mathrm{O}}}=0.319 \mathrm{~nm}$ and $\varepsilon_{\mathrm{C}-\mathrm{O}_{\mathrm{H}_{2} \mathrm{O}}}=0.392 \mathrm{~kJ} / \mathrm{mol}$. In the opacity cases, the $\varepsilon_{\mathrm{C}-\mathrm{O}_{\mathrm{H}_{2} \mathrm{O}}}$ parameter of the Lenndard-Jones potential is tuned to account for an increasing strength of the interaction energy. It should be noticed that the water molecules do not interact with silica atoms in the opacity cases.

In the translucency cases, the van der Waals interactions between silica atoms and the water molecules are described using a Buckingham potential:

$$
U_{i j}\left(r_{i j}\right)=\alpha_{\alpha \beta} \exp \left(-\frac{r_{i j}}{\rho_{\alpha \beta}}\right)-\frac{C_{\alpha \beta}}{r_{i j}^{6}},
$$

where $\alpha_{\alpha \beta}, \rho_{\alpha \beta}$ and $C_{\alpha \beta}$ are adjustable interaction parameters. The parameters are taken from the work of Zambrano et al. ${ }^{53}$ and the work of Hassanali and Singer. ${ }^{52}$ The strength of the interaction energy is systematically increased by modifying the $C_{\alpha \beta}$ parameter of the Buckingham potential between $\mathrm{O}_{\mathrm{H}_{2} \mathrm{O}}$ and $\mathrm{O}_{\mathrm{SiO}_{2}}$ atoms. The values of the parameters are listed in table 1. It should be noticed that only the $C_{\mathrm{O}_{\mathrm{SiO}_{2}}-\mathrm{O}_{\mathrm{H}_{2} \mathrm{O}}}$ parameter is tuned for this set of simulations. Therefore, the parameters for the interactions between Si atoms and $\mathrm{H}_{2} \mathrm{O}$ molecules, and the parameters for the interaction between $\mathrm{C}$ atoms and $\mathrm{H}_{2} \mathrm{O}$ molecules, remain unmodified for all the translucency cases. 


\section{Results and discussion}

\section{Water contact angle}

The strength of the interaction between the coated silica pore and water is characterized by measuring the WCA of a sessile droplet confined inside the pore. The WCA is computed by applying a circular fit to the interfaces of the confined droplets and calculating the tangent of the fit at the CNT surface. Moreover, different degrees of wettability in each scenario are reproduced by systematically modifying the value of the force field parameters. Figure 2 shows the computed WCA for (a) translucent and (b) opacity cases. As presented in this figure, a linear fit is applied to the computed WCAs for each scenario. Note that we employ these linear fits to estimate the WCA in each subsequent case studied here. For a detailed description of the WCA measurements, readers are referred to the Supporting Information.

While we aimed to represent the real material as close as possible, our model is still a simplification that consider a limited number of variables. Thus, in an attempt to maintain simplicity, we have tuned the strength of the interaction between silica and water molecules by only modifying the $C_{\mathrm{O}_{\mathrm{Si}_{2}}-\mathrm{O}_{\mathrm{H}_{2} \mathrm{O}}}$ parameter of the Buckingham potential. We are aware that another alternative to tune the wettability of the coated substrate is to modify the interaction parameters of the $\mathrm{Si}-\mathrm{H}_{2} \mathrm{O}$ interactions, along with the $\mathrm{O}_{\mathrm{SiO}_{2}}-\mathrm{H}_{2} \mathrm{O}$ interactions. Nevertheless, the inclusion of more parameters to be tuned would require an extensive parametric study which is beyond the scope of the present investigation.

\section{Poiseuille Flow}

We investigate the interplay between wetting and slippage by simulating Poiseuille-like flow of water in a silica pore coated by a SWCNT. WCAs between $10^{\circ}$ and $95^{\circ}$ are computed for the translucency and opacity cases. In particular, for the translucency cases, the tuning parameter $C_{\mathrm{O}_{\mathrm{SiO}_{2}}-\mathrm{O}_{\mathrm{H}_{2} \mathrm{O}}}$ ranges from 0.0 to $0.07 \mathrm{~kJ} \mathrm{~nm} / \mathrm{mol}$. On the other hand, the tuning parameter $\epsilon_{\mathrm{C}-\mathrm{O}_{\mathrm{H}_{2} \mathrm{O}}}$ ranges from 0.392 to $0.675 \mathrm{~kJ} / \mathrm{mol}$ for the opacity cases. Note that the 
number of water molecules in each system is chosen to reproduce an internal pressure of 1 bar (see Supporting Information). Fluid flow is generated by imposing a constant acceleration field $\left(F_{e}\right)$ in the axial direction to all water molecules confined inside the pore. The values of $F_{e}$ range from $1.5 \times 10^{11}$ to $6 \times 10^{11} \mathrm{~m} / \mathrm{s}^{2}$, corresponding to pressure gradients between 1.5 and $6.0 \mathrm{bar} / \mathrm{nm}$. Each simulation is conducted for $500 \mathrm{ps}$ in order to reach steady state, and then for another $20 \mathrm{~ns}$ to extract data of the trajectories every $10 \mathrm{ps}$.

In addition to the cases reproducing translucency and opacity, we simulate the flow of water inside a pristine Single Walled CNT (SWCNT) to quantify the effect of the CNT corrugation over water slippage. Such system consists of water confined in a $(39,39)$ CNT, with the same dimensions to the one employed as a coating within the silica pore. The atoms of the CNT are maintained active and coupled to a Berendsen thermostat, with a coupling constant of $0.005 \mathrm{ps}$. In order to avoid large oscillations in the CNT, we employ a second coaxial $(44,44)$ CNT to restrict the movement of the $(39,39)$ CNT. The positions of the atoms in the outer CNT are maintained fixed through all the simulations. Note that the atoms of the outer CNT only interact with the atoms of the inner CNT without direct interaction with the confined water molecules. We employ the results of the filling simulations of the opacity cases to calculate the number of water molecules confined inside the SWCNT. For the SWCNT cases, the values of constant acceleration $F_{e}$ range from $0.2 \times 10^{11}$ to $1.6 \times 10^{11} \mathrm{~m} / \mathrm{s}^{2}$, corresponding to pressure gradients between 0.2 and $1.6 \mathrm{bar} / \mathrm{nm}$.

For each case, the radial velocity profile is computed by employing a cylindrical binning with a radial bin size of $0.3 \mathrm{~nm}$. The slip velocity $\left(u_{s}\right)$, i.e., the fluid velocity at the CNT surface is estimated for each case from a parabolic fit applied to the velocity profiles. Thereafter, the slip length $\left(l_{s}\right)$ is calculated by applying a linear fit to the slip velocity as a function of the applied external field as follows: ${ }^{54}$

$$
l_{s}=\frac{2 m \mu_{0}}{\rho R}
$$

where, $m$ represents the slope of the linear fit, $\mu_{0}$ is the bulk viscosity of the fluid $(0.729$ 
$\mathrm{mPa} \mathrm{s}{ }^{55}$ ), $\rho$ is the overall density of the fluid (total mass of water in the system divided by the available volume inside the pore) and $R$ is the CNT radius corrected to consider the van der Waals size of the carbon atoms $(R=2.4509 \mathrm{~nm})$. From the $l_{s}$, we compute the flow enhancement $(E)$, i.e., the ratio between the flow of water inside the pore $(Q)$ and the no-slip Hagen-Poiseuille flow prediction $\left(Q_{H P}\right)$. Here, the $E$ is estimated as: ${ }^{25}$

$$
E=\frac{Q}{Q_{H P}}=1+\frac{4 l_{s}}{R}
$$

where $Q$ is computed as $Q=\pi R^{2}\left\langle u_{z}\right\rangle$, where $\left\langle u_{z}\right\rangle$ represents the average velocity of water in the axial direction. The computed values of $l_{s}$ for all the cases are presented in Figure 3, along with the calculated $E$. Note that the translucency cases exhibit the lowest $E$, with values ranging from 10 to 50 which indicates that the water flow rate is at least 10 times faster than the one predicted by the no-slip Poiseuille relation. Therefore, our results suggest that a significant reduction in the hydrodynamic loss is expected by implementing a SWCNT as surface coating in a hydrophilic silica nanopore. With regard to the relationship between wettability and slippage, we observe an increase in the $l_{s}$ as the WCA increases for all the scenarios evaluated in this study. Furthermore, we observe that the scaling law $l_{s} \sim\left(180^{\circ}-\mathrm{WCA}\right)^{-2}$ proposed by Huang et. al. ${ }^{56}$ provides a good fit to our results. Nevertheless, the translucency cases exhibit a $l_{s}$ value substantially lower than the value in the corresponding opacity cases. This difference indicates that the mechanism by which water molecules interacts with the pore has a significant influence on the interfacial fluid slippage. Moreover, the SWCNT cases exhibit even larger $l_{s}$, suggesting that the molecular roughness of the surface plays a key role in the phenomenon of fluid slippage, which is not taken into account by the scaling laws that correlate $l_{s}$ and WCA. This breakdown of the scaling laws for liquids on a coated substrate has previously been reported by Ramos-Alvarado et al. ${ }^{57}$ In their work, they compare the hydrodynamic properties of water in contact with two silicon substrates that differ in their crystallographic structure, with and without a monolayer GE as coating. In particular, they propose that the scaling law $l_{s} \sim \delta^{4}$ (also proposed by Huang 
et al. ${ }^{56,58}$ ) provides better quantitative predictions of the slip boundary condition on the studied surfaces. In this scaling law, $\delta$ represents the depletion length, i. e., the thickness of the depletion layer at the water-carbon interface. Following their results, we compute $\delta$ of water confined inside the pore for both wetting scenarios as:

$$
\delta=\int_{0}^{\infty}\left[1-\frac{\rho_{s}(r)}{\rho_{s, b u l k}}-\frac{\rho_{w}(r)}{\rho_{w, b u l k}}\right] d r
$$

where $\rho_{s}$ represents the density of the solid, and $\rho_{w}$ the density of water. The bulk densities $\left(\rho_{w, \text { bulk }}\right.$ and $\left.\rho_{s, b u l k}\right)$ are computed directly from our simulations. In Figure 4 , the $l_{s}$ is presented as a function of the computed $\delta$ for both scenarios. The solid lines in this figure represent a fit to the scaling law $l_{s} \sim \delta^{4}$. For the translucency case, we observe that the results from our simulations fit the values obtained using this scaling law. In contrast, this relationship tends to fail for the opacity scenario. Nevertheless, a better agreement is found by including a constant correction term to the scaling law $\left(l_{s} \sim \delta^{4}+a\right.$, where $a$ represents the constant correction term), as shown in Figure 4 wherein the dashed line depicts a fit to the corrected scaling law. Moreover, a substantial difference between the $l_{s}$ values for both scenarios is still observed, suggesting that the $\delta$ parameter alone is not sufficient to predict the slip boundary condition.

\section{Energy Landscape}

In order to get further insight into the observed difference in the slippage for the opacity and translucency cases, we examine the potential energy landscape for the water molecules inside the coated pore. To this end, we sample the potential energy resultant of the interaction between a single water molecule and the pore atoms (using the correspondent model for each scenario). For the translucency cases, the mean potential energy is computed by summing up the interaction energy between the water molecule and atoms in the CNT and silica walls. Whereas, only the interaction energy between the water molecule and SWCNT 
atoms is taken into account for the opacity cases. Note that for both cases, the CNT wall exhibits the same roughening as a result of the presence of the silica pore wall. The standard deviation of the mean potential energy as a function of the distance between the water molecule $\left(r_{\mathrm{C}_{-} \mathrm{H}_{2} \mathrm{O}}\right)$ and the carbon atoms in the SWCNT is depicted in Figure 5. In general, the translucency cases exhibit higher standard deviations than the opacity cases, indicating that the water interaction with silica increases the corrugation of the potential energy landscape. This dissimilarity can be better appreciated by considering the potential landscape near the surface of the pore. Figure 6 a shows the potential energy landscape at a distance $r_{\mathrm{C}-\mathrm{O}_{\mathrm{H}_{2} \mathrm{O}}}$ of $0.319 \mathrm{~nm}$ for the opacity case with $\varepsilon_{\mathrm{C}-\mathrm{O}_{\mathrm{H}_{2} \mathrm{O}}}=0.45 \mathrm{~kJ} / \mathrm{mol}$. Moreover, Figure $6 \mathrm{~b}$ shows the potential energy landscape at the same distance for the translucency case with $C_{\mathrm{O}_{\mathrm{SiO}_{2}}-\mathrm{O}_{\mathrm{H}_{2} \mathrm{O}}}=0.01 \mathrm{~kJ} \mathrm{~nm}^{6} / \mathrm{mol}$. It should be noticed that the interaction parameters for both cases reproduce a WCA of approximately $78^{\circ}$. In the opacity case, the potential landscape displays hexagonal periodic features inherited from the crystallographic features of the CNT, whereas these hexagonal features are not present in the translucency case. In addition, it can be appreciated that the opacity case exhibits a much smoother potential than the translucency case, in line with the difference in the standard deviations of the potentials shown in Figure 5. Hence, in agreement with previous studies that associate surface roughness and slipagge, ${ }^{22,28,29,39,59-64}$ we link the observed disparity in the $l_{s}$ values computed for both scenarios, to a significant increase of the corrugation of the energy landscape induced by the roughness of the underlying silica substrate. Moreover, the quantitative disagreement between the scaling laws ${ }^{57,58}$ and our results for opacity cases, suggests that a term that includes information about the roughness of the underlying substrate is required to accurately predict the water slippage on surfaces coated by atomically thin materials like CNTs, graphene or hexagonal boron nitride layers. 


\section{Conclusions}

In this work, MD and NEMD simulations are performed to probe the effect of a SWCNT coating on the transport properties of water confined in a hydrophilic silica nanopore. To this end, an atomistic model of a cylindrical silica nanopore, internally coated by a $(39,39)$ SWCNT, is characterized. The interaction between water molecules and the coated pore is described based on two possible scenarios: translucency and opacity to wettability of the SWCNT coating. In the translucency scenario, water molecules directly interact with the SWCNT atoms and the atoms in the underlying substrate. In contrast, in the opacity scenario water molecules interact exclusively with the SWCNT atoms while silica and CNT atoms interact with each other. In both scenarios, the interactions between water and solid walls are characterized by measuring the WCA and the potential energy landscape within the nanopore. Moreover, the effect of wettability on the interfacial slippage is systematically studied by simulating Poiseuille like-flow of water, under different strengths of the water-pore interaction. Our results confirm the dependence of the slip length on the WCA. Nevertheless, different fluid slip lengths are found for the two scenarios considered, suggesting that scaling laws that correlate wetting and slippage cannot accurately predict the slip boundary condition at the nanoscale without considering the surface energy corrugation felt by confined water. This disparity in slippage can be explained by the different surface energy corrugation induced by the roughness of the underlying substrate as a result of directly taking into account the silica-water interaction in the translucency scenario. Despite this different behaviors of the evaluated wettability models, our results indicate that a significant flow enhancement is obtained by employing a SWCNT as coating in hydrophilic nanopores. This insight can assist design of efficient nanofluidic devices and further development of nanomembranes for fast water transport and molecular sieving. 


\section{Acknowledgement}

E. Wagemann thanks financial support from Centro CRHIAM Conicyt/Fondap Project 15130015 (Chile). This research was partially funded by CONICYT under FONDECYT project No 11130559. The authors thank computational support from the Departments of Physics and Mechanical Engineering at the Technical University of Denmark and from National Laboratory for High Performance Computing (NLHPC). H. Zambrano acknowledges support from the Universidad Técnica Federico Santa María through a DGIIP research grant.

\section{Supporting Information Available}

The following files are available free of charge.

- supporting.pdf: Interaction potentials and models, Pore characterization, Hydrodynamics of water confined within the pore, Potential energy landscape.

This material is available free of charge via the Internet at http://pubs.acs.org/.

\section{References}

(1) Biswas, A.; Bayer, I. S.; Biris, A. S.; Wang, T.; Dervishi, E.; Faupel, F. Advances in top-down and bottom-up surface nanofabrication: Techniques, applications \& future prospects. J. Coll. Interface Sci. 2012, 170, 2-27.

(2) Wang, C.; Nam, S.-W.; Cotte, J. M.; Jahnes, C. V.; Colgan, E. G.; Bruce, R. L.; Brink, M.; Lofaro, M. F.; Patel, J. V.; Gignac, L. M. et al. Wafer-scale integration of sacrificial nanofluidic chips for detecting and manipulating single DNA molecules. Nat. Commun. 2011, 8, 1-9.

(3) Yu, H.-D.; Regulacio, M. D.; Ye, E.; Han, M.-Y. Chemical routes to top-down nanofabrication. Chem. Soc. Rev. 2013, 42, 6006-6018. 
(4) Duan, C.; Wang, W.; Xie, Q. Fabrication of nanofluidic devices. Biomicrofluidics 2013, ᄀ, 1-41.

(5) Lin, X.; Yang, Q.; Ding, L.; Su, B. Ultrathin Silica Membranes with Highly Ordered and Perpendicular Nanochannels for Precise and Fast Molecular Separation. ACS Nano. 2015, 9, 11266-11277.

(6) Zhao, C.; Lu, J.; Hou, J.; Li, X.; Wang, J.; Jiang, L.; Wang, H.; Zhang, H. Bioinspired Self-Gating Nanofluidic Devices for Autonomous and Periodic Ion Transport and Cargo Release. Adv. Funct. Mater. 2019, 29, 1-8.

(7) Ulissi, Z. W.; Shimizu, S.; Lee, C. Y.; Strano, M. S. Carbon Nanotubes as Molecular Conduits: Advances and Challenges for Transport through Isolated Sub-2 nm Pores. J. Phys. Chem. Lett. 2011, 2, 2892-2896.

(8) Radha, B.; Esfandiar, A.; Wang, F.; Rooney, A.; Gopinadhan, K.; Keerthi, A.; Mishchenko, A.; Janardanan, A.; Blake, P.; Fumagalli, L. et al. Molecular transport through capillaries made with atomic-scale precision. Nature 2016, 538, 222-225.

(9) Jung, W.; Kim, J.; Kim, S.; Park, H. G.; Jung, Y.; Han, C.-S. A Novel Fabrication of $3.6 \mathrm{~nm}$ High Graphene Nanochannels for Ultrafast Ion Transport. Advanced Mat. 2008, 29, 1-7.

(10) Thomas, M.; Corry, B.; Hilder, T. A. What have we learnt about the mechanisms of rapid water transport, ion rejection and selectivity in nanopores from molecular simulation. Small 2014, 10, 1453-1465.

(11) Goh, K.; Karahan, H. E.; Wei, L.; Bae, T.-H.; Fane, A. G.; Wang, R.; Chen, Y. Carbon nanomaterials for advancing separation membranes: A strategic perspective. Carbon 2016, 109, 694-710. 
(12) Chin, C. D.; Linder, V.; Sia, S. K. Lab-on-a-chip devices for global health: Past studies and future opportunities. Lab Chip 2007, 7, 41-57.

(13) Sanhai, W. R.; Sakamoto, J. H.; Canady, R.; Ferrari, M. Seven challenges for nanomedicine. Nature Nanotechnol. 2008, 3, 242.

(14) Wanunu, M. Nanopores: A journey towards DNA sequencing. Phys. Life Rev. 2012, 9, $125-158$.

(15) Bocquet, L.; Charlaix, E. Nanofluidics, from bulk to Interfaces. Chem. Soc. Rev. 2010, 39, 1073-1095.

(16) Farimani, A. B.; Aluru, N. Spatial diffusion of water: from Fickian to ballistic motion. J. Phys. Chem. B 2011, 115, 12145-12149.

(17) Kohler, M. H.; Bordin, J. R.; de Matos, C. F.; Barbosa, M. C. Water in nanotubes: The surface effect. Chem. Eng. Sci. 2019, 203, 54-67.

(18) Alibakhshi, M. A.; Xie, Q.; Li, Y.; Duan, C. Accurate measurement of liquid transport through nanoscale conduits. Sci. Rep. 2016, 6, 1-8.

(19) Bati, A. S. R.; Yu, L.; Batmunkh, M.; Shapter, J. G. Synthesis, purification, properties and characterization of sorted single-walled carbon nanotubes. Nanoscale 2018, 10 , $22087-22139$.

(20) Corry, B. Designing carbon nanotube membranes for efficient water desalination. J. Phys. Chem. B 2008, 112, 1427-1434.

(21) Majumder, M.; Chopra, N.; Andrews, R.; Hinds, B. J. Nanoscale hydrodynamics: enhanced flow in carbon nanotubes. Nature 2005, 438, 44-44.

(22) Falk, K.; Sedlmeier, F.; Joly, L.; Netz, R. R.; Bocquet, L. Molecular origin of fast water transport in carbon nanotube membranes: superlubricity versus curvature dependent friction. Nano Lett. 2010, 10, 4067-4073. 
(23) Thomas, J. A.; McGaughey, A. J. Reassessing fast water transport through carbon nanotubes. Nano Lett. 2008, 8, 2788-2793.

(24) Holt, J. K.; Park, H. G.; Wang, Y.; Stadermann, M.; Artyukhin, A. B.; Grigoropoulos, C. P.; Noy, A.; Bakajin, O. Fast mass transport through sub-2-nanometer carbon nanotubes. Science 2006, 312, 1034-1037.

(25) Kannam, S. K.; Todd, B.; Hansen, J. S.; Daivis, P. J. How fast does water flow in carbon nanotubes? J. Chem. Phys. 2013, 138, 094701.

(26) Whitby, M.; Quirke, N. Fluid flow in carbon nanotubes and nanopipes. Nature Nanotechnol. 2007, 2, 87-94.

(27) Lopez-Lorente, A. I.; Simonet, B. M.; Valcarcel, M. The potential of carbon nanotube membranes for analytical separations. Anal. Chem. 2010, 82, 5399-5407.

(28) Joseph, S.; Aluru, N. Why are carbon nanotubes fast transporters of water? Nano Lett. 2008, 8, 452-458.

(29) Tocci, G.; Joly, L.; Michaelides, A. Friction of water on graphene and hexagonal boron nitride from ab initio methods: very different slippage despite very similar interface structures. Nano Lett. 2014, 14, 6872-6877.

(30) Li, Z.; Wang, Y.; Kozbial, A.; Shenoy, G.; Zhou, F.; McGinley, R.; Ireland, P.; Morganstein, B.; Kunkel, A.; Surwade, S. P. et al. Effect of airborne contaminants on the wettability of supported graphene and graphite. Nature Mat. 2013, 12, 925-931.

(31) Rafiee, J.; Mi, X.; Gullapalli, H.; Thomas, A. V.; Yavari, F.; Shi, Y.; Ajayan, P. M.; Koratkar, N. A. Wetting transparency of graphene. Nature Mat. 2012, 11, 217-222.

(32) Shih, C.-J.; Wang, Q. H.; Lin, S.; Park, K.-C.; Jin, Z.; Strano, M. S.; Blankschtein, D. Breakdown in the wetting transparency of graphene. Phys. Rev. Lett. 2012, 109, 176101. 
(33) Shih, C.-J.; Strano, M. S.; Blankschtein, D. Wetting translucency of graphene. Nature Mat. 2013, 12, 866-869.

(34) Raj, R.; Maroo, S. C.; Wang, E. N. Wettability of Graphene. Nano Lett. 2013, 13, 1509-1515.

(35) Hung, S.-W.; Hsiao, P.-Y.; Chen, C.-P.; Chieng, C.-C. Wettability of Graphene-Coated Surface: Free Energy Investigations Using Molecular Dynamics Simulation. J. Phys. Chem. C 2015, 119, 8103-8111.

(36) Mirsaidov, U.; Mokkapati, V. R. S. S.; Bhattacharya, D.; Andersen, H.; Bosman, M.; Ozyilmaz, B.; Matsudaira, P. Scrolling graphene into nanofluidic channels. Lab Chip 2013, 13, 2874-2878.

(37) Hong, G.; Han, Y.; Schutzius, T. M.; Wang, Y.; Pan, Y.; Hu, M.; Jie, J.; Sharma, C. S.; Muller, U.; Poulikakos, D. On the mechanism of hydrophilicity of graphene. Nano Lett. 2016, 16, 4447-4453.

(38) Ashraf, A.; Wu, Y.; Wang, M. C.; Yong, K.; Sun, T.; Jing, Y.; Haasch, R. T.; Aluru, N. R.; Nam, S. Doping-Induced Tunable Wettability and Adhesion of Graphene. Nano Lett. 2016, 16, 4708-4712.

(39) Wagemann, E.; Oyarzua, E.; Walther, J. H.; Zambrano, H. A. Slip divergence of water flow in graphene nanochannels: the role of chirality. Phys. Chem. Chem. Phys. 2017, $19,8646-8652$.

(40) Werder, T.; Walther, J. H.; Jaffe, R. L.; Halicioglu, T.; Noca, F.; Koumoutsakos, P. Molecular dynamics simulation of contact angles of water droplets in carbon nanotubes. Nano Lett. 2001, 1, 697-702.

(41) Werder, T.; Walther, J. H.; Jaffe, R. L.; Halicioglu, T.; Koumoutsakos, P. On the 
water-graphite interaction for Use in MD simulations of graphite and carbon nanotubes. J. Phys. Chem. B 2003, 10\%, 1345-1352.

(42) Zambrano, H. A.; Walther, J. H.; Koumoutsakos, P.; Sbalzarini, I. F. Thermophoretic Motion of Water Nanodroplets Confined Inside Carbon Nanotubes. Nano Lett. 2009, 9, 66-71.

(43) Zambrano, H. A.; Vásquez, N.; Wagemann, E. Wall embedded electrodes to modify electroosmotic flow in silica nanoslits. Phys. Chem. Chem. Phys. 2016, 18, 1202-1211.

(44) Oyarzua, E.; Walther, J. H.; Mejia, A.; A., Z. H. Early regimes of water capillary flow in slit silica nanochannels. Phys. Chem. Chem. Phys. 2015, 17, 14731-14739.

(45) Walther, J. H.; Ritos, K.; Cruz-Chu, E. R.; Megaridis, C. M.; Koumoutsakos, P. Barrier to Superfast Water Transport in Carbon Nanotube Membranes. Nano Lett. 2013, 13, $1910-1914$.

(46) Berendsen, H. J. C.; Postma, J. P. M.; van Gunsteren, W. F.; DiNola, A.; Haak, J. R. Molecular dynamics with coupling to an external bath. J. Chem. Phys. 1984, 81, 36843684 .

(47) Essmann, U.; Perera, L.; Berkowitz, M. L.; Darden, T.; Lee, H.; Pedersen, L. G. A smooth particle mesh Ewald method. J. Chem. Phys. 1995, 103, 8577-8593.

(48) Berendsen, H. J. C.; Grigera, J. R.; Straatsma, T. P. The missing term in effective pair potentials. J. Phys. Chem. 1987, 91, 6269-6271.

(49) Walther, J. H.; Jaffe, R.; Halicioglu, T.; Koumoutsakos, P. Carbon nanotubes in water: Structural characteristics and energetics. J. Phys. Chem. B 2001, 105, 9980-9987.

(50) Zhang, Z.; Li, T. A molecular mechanics study of morphologic interaction between graphene and $\mathrm{Si}$ nanowires on a $\mathrm{SiO}_{2}$ substrate. J Nanomater. 2011, 2011, 1-7. 
(51) Guissani, Y.; Guillot, B. A numerical investigation of the liquid-vapor coexistence curve of silica. J. Chem. Phys. 1996, 104, 7633-7644.

(52) Hassanali, A. A.; Singer, S. J. Model for the water-amorphous silica interface: The undissociated surface. J. Phys. Chem. B 2007, 111, 11181-11193.

(53) Zambrano, H.; Walther, J. H.; Jaffe, R. Molecular dynamics simulations of water on a hydrophilic silica surface at high air pressures. J. Mol. Liq. 2014, 198, 107-113.

(54) Kannam, S. K.; Todd, B.; Hansen, J. S.; Daivis, P. J. Slip length of water on graphene: Limitations of non-equilibrium molecular dynamics simulations. J. Chem. Phys. 2012, $136,024705$.

(55) González, M. A.; Abascal, J. L. The shear viscosity of rigid water models. J. Chem. Phys. 2010, 132, 096101.

(56) Huang, D. M.; Sendner, C.; Horinek, D.; Netz, R. R.; Bocquet, L. Water slippage versus contact angle: a quasiuniversal relationship. Phys. Rev. Lett. 2008, 101, 226101.

(57) Ramos-Alvarado, B.; Kumar, S.; Peterson, G. Wettability transparency and the quasiuniversal relationship between hydrodynamic slip and contact angle. Appl. Phys. Lett. 2016, 108, 074105 .

(58) Sendner, C.; Horinek, D.; Bocquet, L.; Netz, R. R. Interfacial water at hydrophobic and hydrophilic surfaces: Slip, viscosity, and diffusion. Langmuir 2009, 25, 10768-10781.

(59) Wei, X.; Luo, T. Effects of Electrostatic Interaction and Chirality on the Friction Coefficient of Water Flow Inside Single-Walled Carbon Nanotubes and Boron Nitride Nanotubes. J. Phys. Chem. C 2018, 122, 5131-5140.

(60) Sega, M.; Sbragaglia, M.; Biferale, L.; Succi, S. Regularization of the slip length divergence in water nanoflows by inhomogeneities at the Angstrom scale. Soft Matter 2013, 9, 8526-8531. 
(61) Wang, F.-C.; Zhao, Y.-P. Slip boundary conditions based on molecular kinetic theory: The critical shear stress and the energy dissipation at the liquid-solid interface. Soft Matter 2011, 7, 8628-8634.

(62) Govind Rajan, A.; Strano, M. S.; Blankschtein, D. Liquids with Lower Wettability Can Exhibit Higher Friction on Hexagonal Boron Nitride: The Intriguing Role of Solid-Liquid Electrostatic Interactions. Nano Lett. 2019, 19, 1539-1551.

(63) Cao, W.; Huang, L.; Ma, M.; Lu, L.; Lu, X. Water in Narrow Carbon Nanotubes: Roughness Promoted Diffusion Transition. The Journal of Physical Chemistry C 2018, 122, 19124-19132.

(64) Galea, T.-M.; Attard, P. Molecular dynamics study of the effect of atomic roughness on the slip length at the fluid- solid boundary during shear flow. Langmuir 2004, 20, $3477-3482$.

\section{Figures and tables}

Table 1: Silica-water interaction parameters.

\begin{tabular}{|c|c|c|c|}
\hline Pair & $C_{\alpha \beta}\left(\mathrm{kJ} \mathrm{nm}^{6} / \mathrm{mol}\right)$ & $\alpha_{\alpha \beta}(\mathrm{kJ} / \mathrm{mol})$ & $\rho_{\alpha \beta}\left(\mathrm{nm}^{-1}\right)$ \\
\hline $\mathrm{O}_{\mathrm{SiO}_{2}}-\mathrm{H}_{\mathrm{H}_{2} \mathrm{O}}{ }^{52}$ & 0.0 & $6.8307 \times 10^{3}$ & 32.6584 \\
$\mathrm{O}_{\mathrm{SiO}_{2}}-\mathrm{O}_{\mathrm{H}_{2} \mathrm{O}}{ }^{52}$ & tuned & $2.7401 \times 10^{5}$ & 31.4355 \\
$\mathrm{Si}_{-} \mathrm{O}_{\mathrm{H}_{2} \mathrm{O}}{ }^{53}$ & 0.0240 & $1.0130 \times 10^{5}$ & 25.0 \\
\hline
\end{tabular}




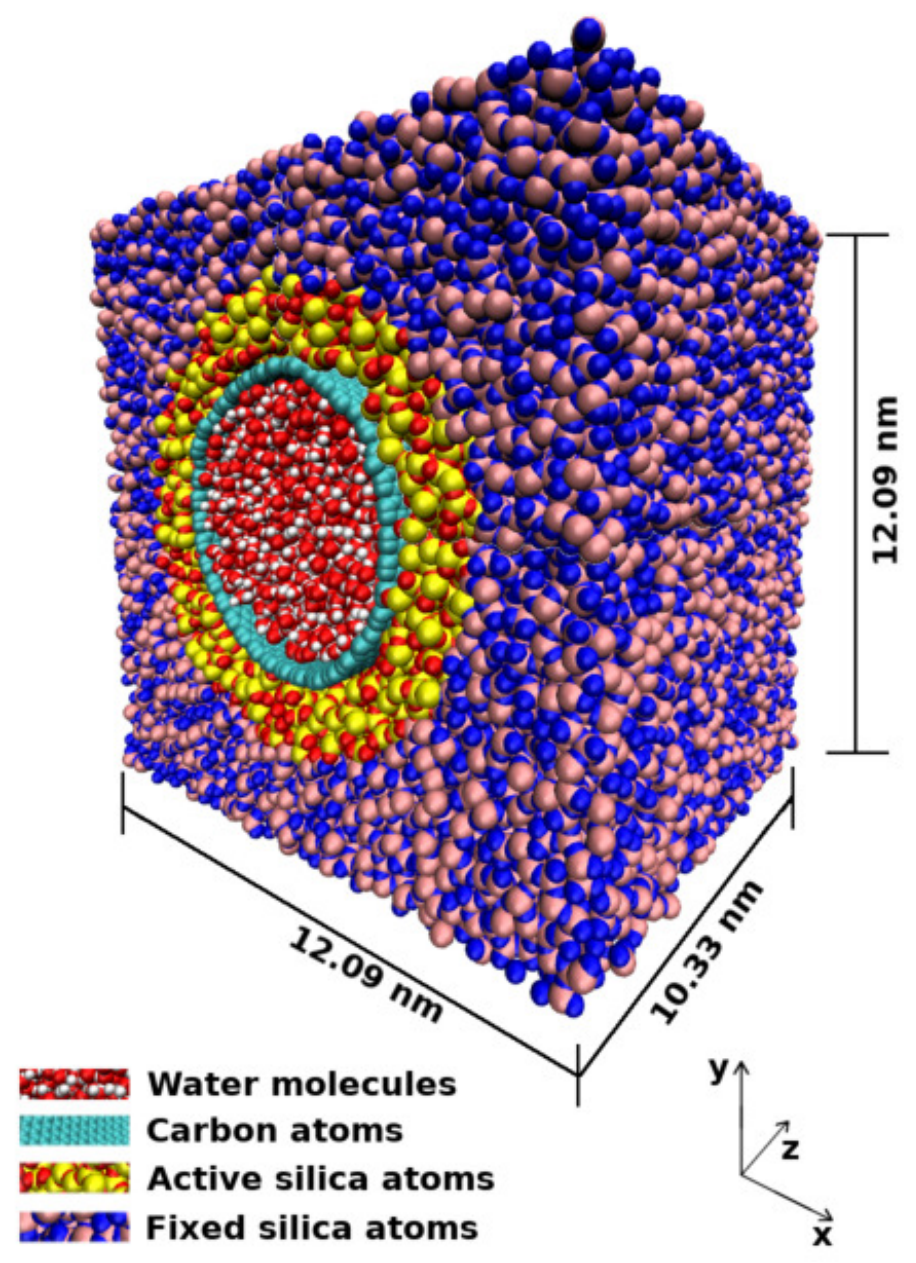

Figure 1: Snapshot of the studied system. An amorphous silica nanopore is internally coated by a $(39,39)$ Armchair CNT. Water is confined inside the CNT. The position of the silica atoms whose radial distance to the CNT is larger than $1.5 \mathrm{~nm}$ is maintained fixed. Heat is extracted by coupling the carbon and internal silica atoms to a Berendsen Thermostat. 
(a)

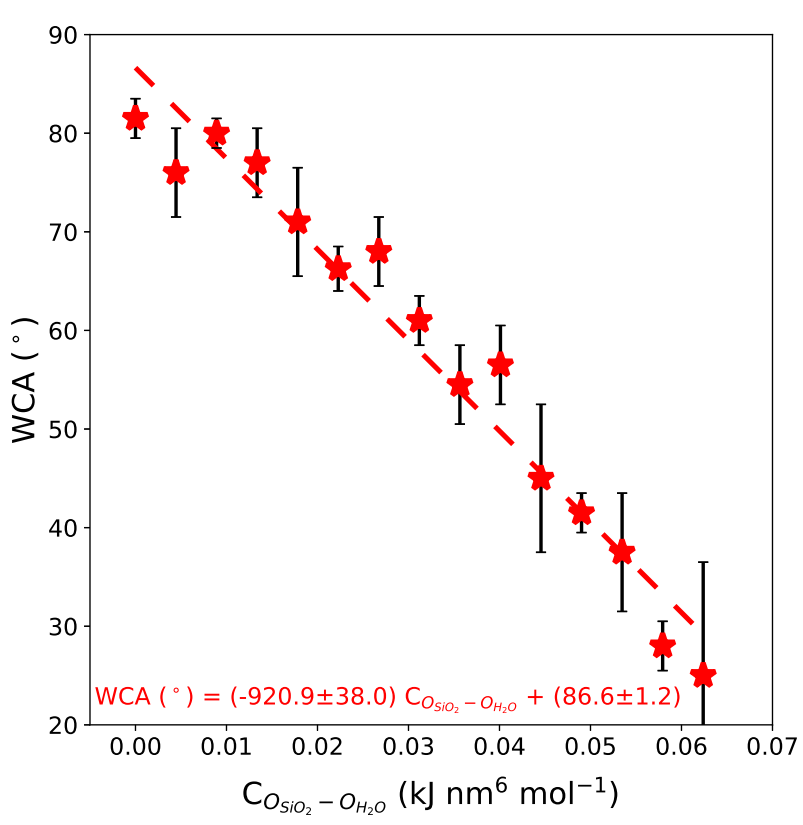

(b)

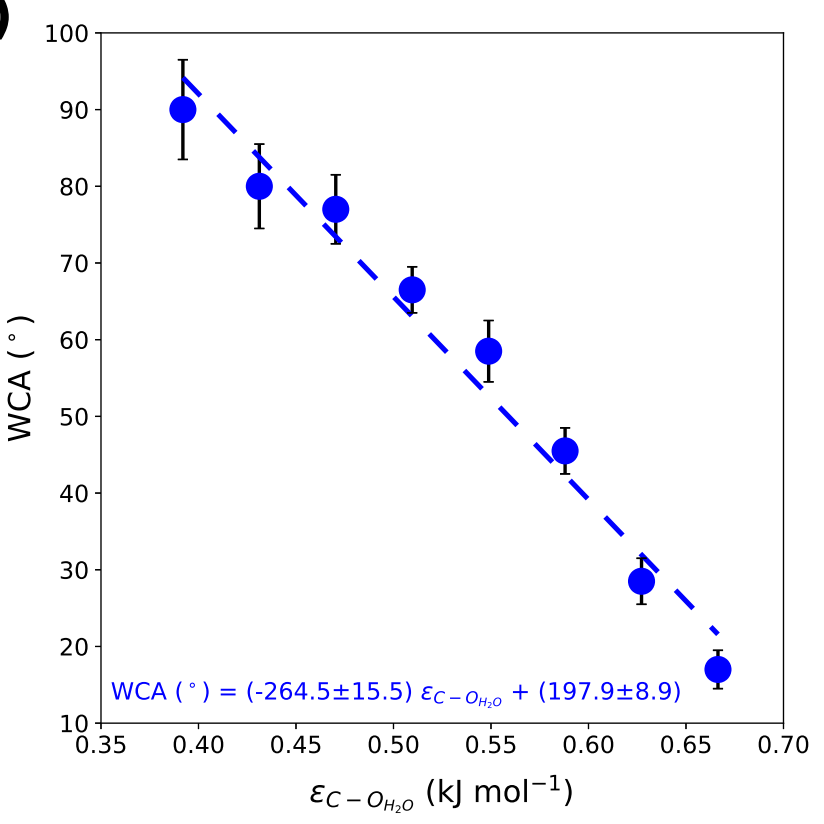

Figure 2: Calculated water contact angles in the CNT coated silica pore for a) the translucency and b) opacity cases. The dashed lines represent a linear fit applied to the computed WCAs. 


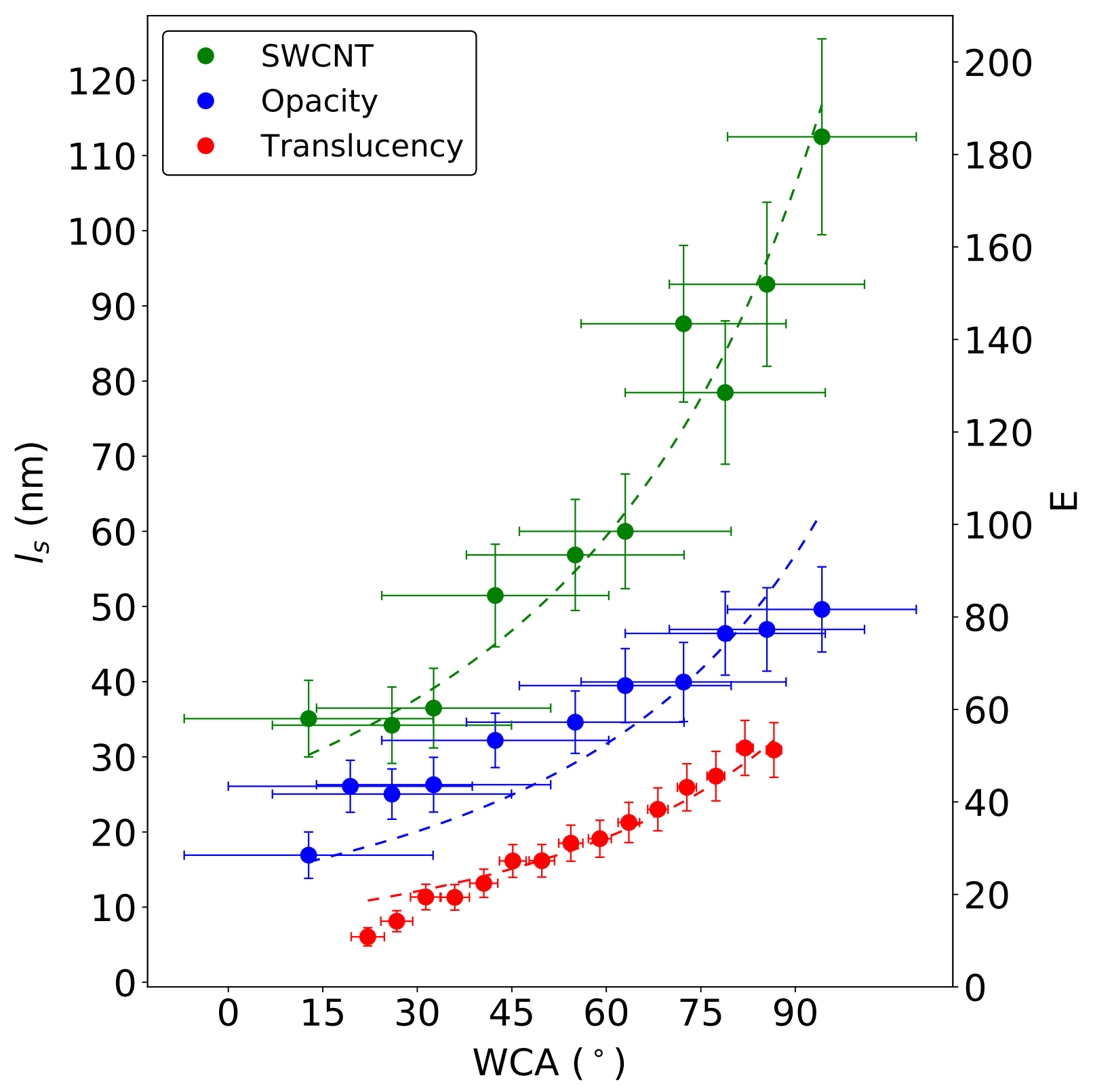

Figure 3: Calculated flow enhancement and slip lengths for all the studied cases of water flowing inside the CNT coated silica pore and the cases of water flow inside the SWCNT. The dashed lines represent a fit to the scaling law $l_{s} \sim\left(180^{\circ}-\mathrm{WCA}\right)^{-2}$. An increase of $E$ and $l_{s}$ is observed with the increase of WCAs for all the studied cases. 


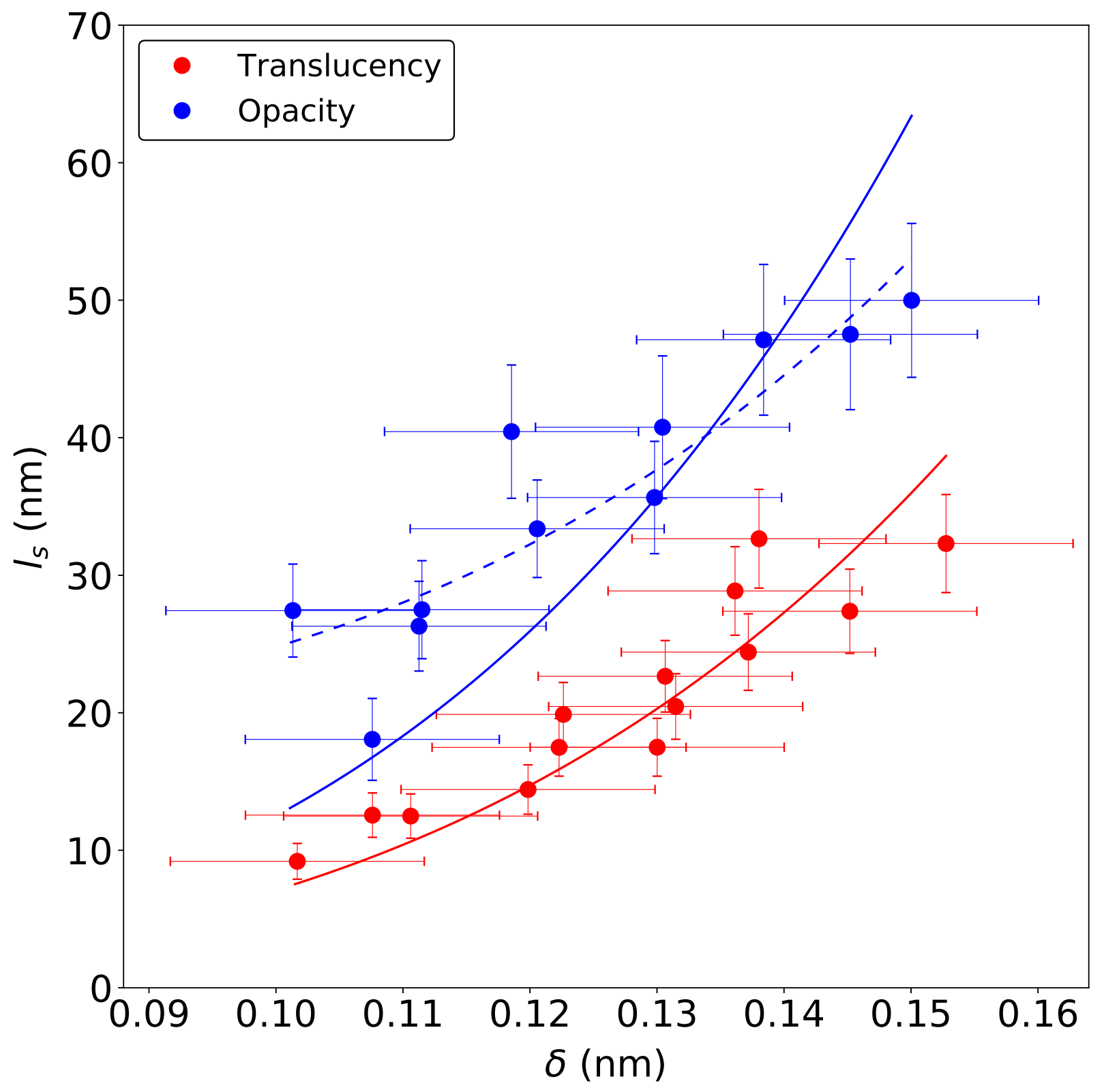

Figure 4: Slip length $l_{s}$ as a function of depletion length $\delta$ for the translucency and opacity cases. The solid lines represent a fit to the scaling law $l_{s} \sim \delta^{4}$. The dashed line represents a fit to $l_{s} \sim \delta^{4}+a$, where $a$ is a correction term. 


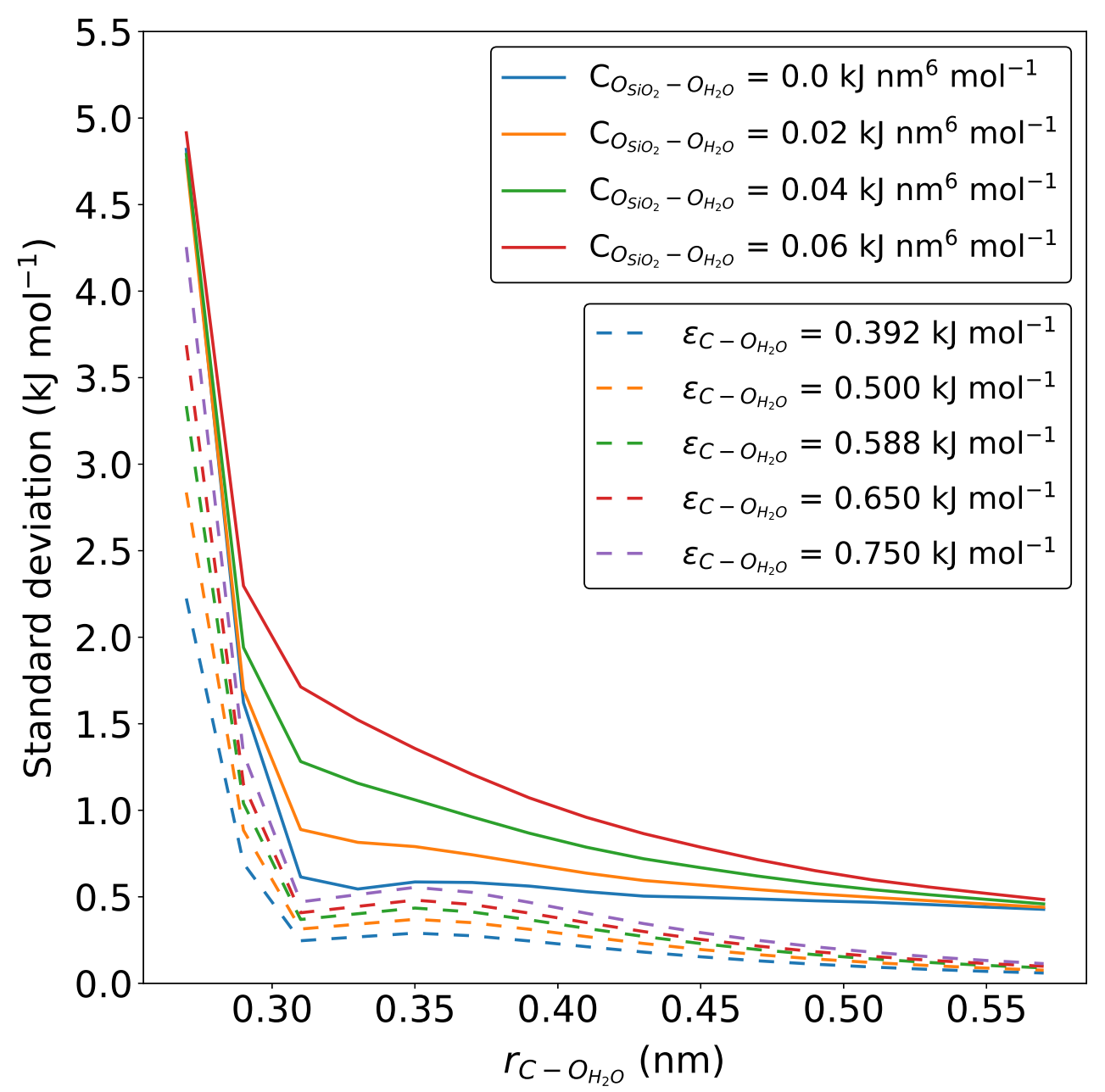

Figure 5: Standard deviation of the calculated potential energy between a single water molecule and the CNT coated silica pore as a function of the distance between the CNT and the water molecule $\left(r_{\mathrm{C}-\mathrm{O}_{\mathrm{H}_{2} \mathrm{O}}}\right)$. In general, the translucency cases (full lines) present larger standard deviations than the opaque cases (dashed), indicating larger surface energy corrugation. 
(a)

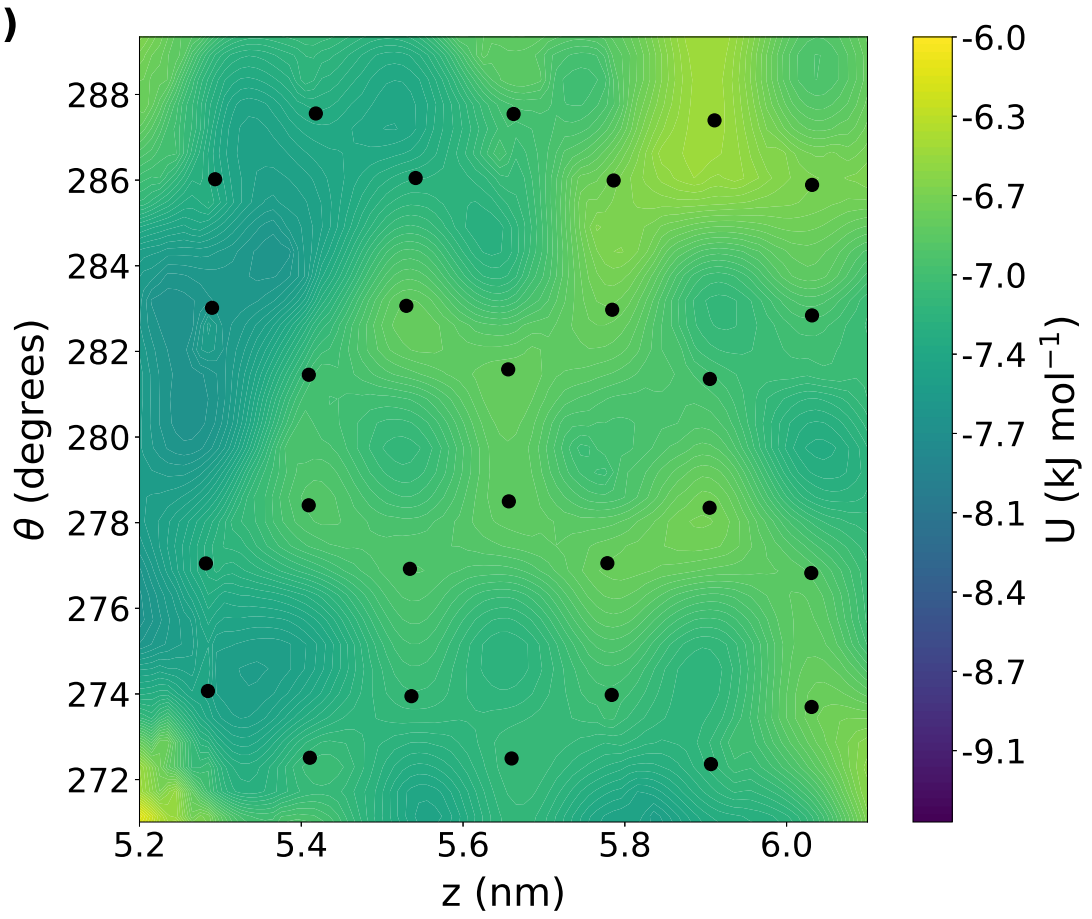

(b)

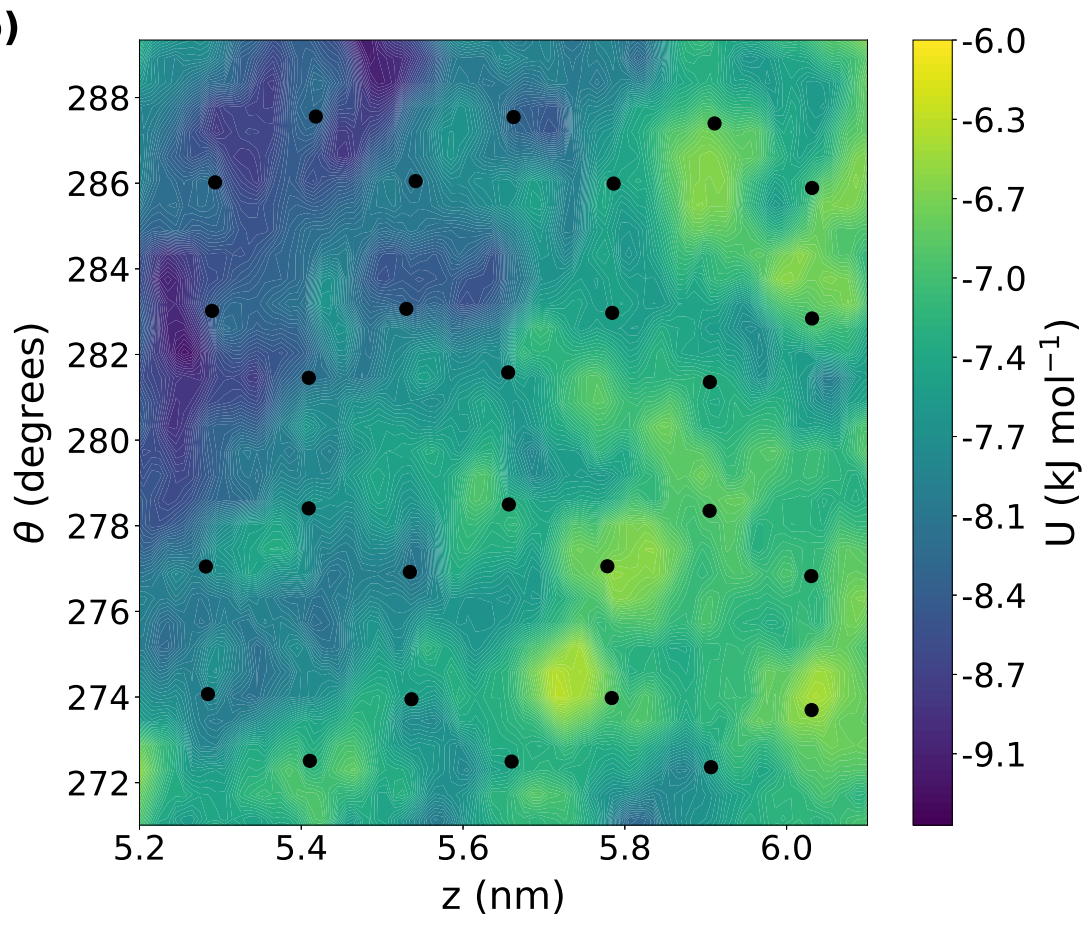

Figure 6: Potential energy landscape at a distance $r_{\mathrm{C}_{-} \mathrm{O}_{2} \mathrm{O}}$ of $0.319 \mathrm{~nm}$ for a) the opacity case with a $\varepsilon_{\mathrm{C}-\mathrm{O}_{\mathrm{H}_{2} \mathrm{O}}}$ of $0.45 \mathrm{~kJ} / \mathrm{mol}$ and b) the translucency case with a $C_{\mathrm{O}_{\mathrm{SiO}_{2}}-\mathrm{O}_{\mathrm{H}_{2} \mathrm{O}}}$ of 0.01 $\mathrm{kJ} \mathrm{nm}^{6} / \mathrm{mol}$. The $\theta$ axis represents circumferential variation and the $z$ axis represents axial variation. The black dots represent the position of the carbon atoms of the CNT. 


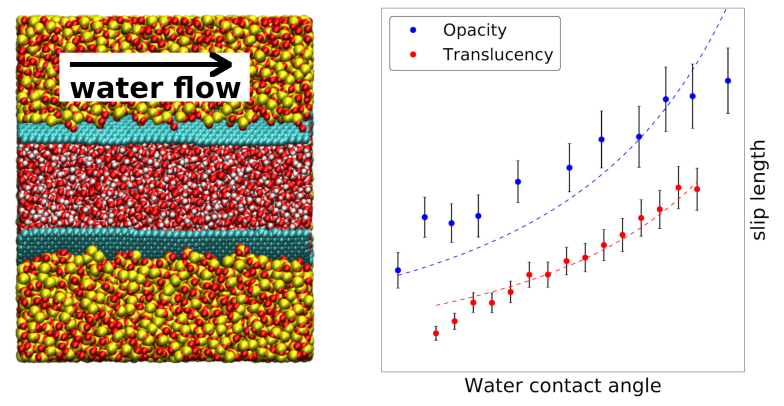

TOC graphic. 